\title{
Dynamisme et diversité
}

\section{Dynamisme and diversity}

\section{J.C. Debongnie}

C) Springer-Verlag France 2010

Nkosi Sikelel'i Africa : Dieu bénisse l'Afrique.

Antienne sud-africaine d'actualité sportive.

Dynamisme... et enthousiasme, telles sont les impressions que donne la coupe du monde en Afrique du Sud. Beaucoup doutaient de la possibilité d'organiser en toute sécurité un événement d'une telle ampleur en Afrique. Bravo à l'Afrique $\mathrm{du}$ Sud. Dynamisme... et enthousiasme, telles sont les impressions que laissent notre association et son journal (le $J A H G$ ) qui en est le reflet. Beaucoup doutaient de la possibilité d'organiser un congrès annuel et encore plus de nourrir un journal! Les $13^{\text {es }}$ Journées viennent d'avoir lieu avec succès à Tunis. Le $J A H G$ a reçu en un peu moins de deux ans plus de 200 soumissions d'articles. Enfin, l'association a lancé un nouveau type de projet commun : un observatoire dont le premier thème est l'hémorragie digestive. En quelques mois, près de 800 observations ont été colligées... et continuent d'affluer. Que tous ceux qui ont soutenu et continuent de soutenir ces projets soient remerciés.

Diversité ! La coupe du monde montre la diversité d'approche des équipes, la différence entre différents publics : la joie africaine s'exprime dans les vuvuzela qui, dans un premier temps, irritent les oreilles d'autres continents. La diversité des pays africains est reflétée dans l'évolution des pays dont 17 fêtent leurs 50 ans d'indépendance (dont le Congo). La diversité d'approche est aussi présente dans nos congrès : l'attitude classique est une coordination régulière entre pays organisateur et comité scientifique, intégrant une participation d'orateurs de pays d'Europe financés par leurs sociétés scientifiques, considérant l'association comme co-organisateur. À Tunis (et à Alger), le comité scientifique est en retrait ..., mais aussi les sociétés scientifiques européennes qui comprennent mal les raisons de financer des orateurs sans participer vraiment. Pourquoi pas ? Si le pays organisateur doit laisser une place à l'association, l'association ne doit-elle pas admettre l'expression de dynamismes particuliers. Place à la diversité !

Et le $J A H G$ ? À Fez et à Yaoundé, des ateliers de rédaction ont eu beaucoup de succès... et ont peut-être contribué à la naissance de nouveaux rédacteurs d'articles. À Tunis, cette formule n'était pas souhaitée, ce qui n'a pas permis la participation officielle du $J A H G$ - pourtant noyau et hautparleur de l'association. D'autres formules de participation doivent être possibles et sont à imaginer : interview des organisateurs, écriture ou recueil d'articles sur place, etc.

Le $J A H G$ et sa rédaction souhaitent participer activement à chaque congrès, suivant une formule à créer chaque fois. Place à la diversité !

Vive le dynamisme... mais aussi vive la diversité.

J.C. Debongnie $(\square)$

e-mail : jc.debongnie@clinique-saint-pierre.be 